\title{
Fundamental Characteristics of a Laser Ablation Microthruster
}

\author{
By Hiroyuki Kolzumi, ${ }^{1)}$ Takayoshi Inoue, ${ }^{1)}$ Kimiya KomuraSAKI ${ }^{2)}$ and Yoshihiro ARAKAwA ${ }^{1)}$ \\ ${ }^{1)}$ Department of Aeronautics and Astronautics, University of Tokyo, Tokyo, Japan \\ ${ }^{2)}$ Department of Advanced Energy, University of Tokyo, Tokyo, Japan
}

(Received June 5th, 2006)

\begin{abstract}
The fundamental characteristics of a laser ablation microthruster were investigated for a $10 \mathrm{~kg}$-class microspacecraft. A single-shot impulse measurement was performed using a thrust stand on which a prototype thruster was installed and the associate ablated mass was estimated from the pressure increase in the space chamber. The best performance of several polymer materials was obtained using polyvinylchloride as the propellant. More heavily carbon doped polyvinylchloride showed higher performance, which means absorption length has a large effect on performance. The intensity of the laser beam on the ablation material was changed using constant laser power, and it was shown that intensity had little effect on the performance. This qualitative behavior agreed with the results of a simple thermal analysis. Mass spectroscopy of the ablation plume showed that the dominant reaction was dehydrochlorination in the range of 470 to $640 \mathrm{~K}$, and the low-temperature reaction resulted in the best performance for polyvinylchloride.
\end{abstract}

Key Words: Microthruster, Microspacecraft, Laser Ablation, Diode Laser

\section{Introduction}

In recent years, microspacecraft have attracted a lot of attention. Research and development of spacecraft, which had been directed to a single large device, have been divided into large and small units. The motivation behind this is to reduce launch and development costs and the development period of spacecraft. In addition, constellation flights of microspacecraft would expand the variety of missions.

The National Aeronautics and Space Administration (NASA) classifies microspacecraft into three classes: Class I, II, and III. ${ }^{1)}$ These microspacecraft classes distinguish themselves by their mass, power, and size ranges. Class I microspacecraft are defined as mass: 5-20 kg, power: 10 $20 \mathrm{~W}$, and scale: about $0.5 \mathrm{~m}$. Class II microspacecraft are defined as mass: $1-5 \mathrm{~kg}$, power: about $1 \mathrm{~W}$, and scale: about $0.1 \mathrm{~m}$. Class III microspacecraft are even smaller spacecraft, defined as mass: less than $1 \mathrm{~kg}$, power: $1 \mathrm{~W}$, and scale: $0.1 \mathrm{~m}$. Class I microspacecraft are supposed to be integrated by conventional components. On the other hand, Class II and III microspacecraft will require further miniaturization and new developments of all components. One of these components is the propulsion system; namely the microthruster. In general, propulsion systems require large weight and size due to the propellant tank, and much further miniaturization is required. However, most state-of-the-art thrusters are not allowed even for Class I microspacecraft, ${ }^{1-4)}$ and Class II and III microspacecraft require entirely new types of thrusters.

A diode laser ablation thruster was proposed by Phipps, C. and Luke, J. as a novel thruster for microspacecraft. ${ }^{5,6)}$ The laser beam from a diode laser is focused on a solid propellant and gas is emitted by laser ablation, producing thrust. Diode lasers work using low voltage, and do not required high voltage. These characteristics are suitable for miniaturization and enable the thruster to be installed on Class I and II microspacecraft.

Thrust produced by laser ablation is generally very small, on the micro-Newton order, and can be controlled by the laser pulse width. Thrust control on the micro-Newton order is necessary for accurate formation flying or keeping formation with the long dead band time of microspacecraft. Miniaturized cold gas thrusters, another microthruster candidate, cannot provide such a small impulse (minimum impulse is usually more than $1 \mathrm{mNs}$ ). In addition, the laser ablation thruster will be more attractive when combined with pyrotechnic laser ignition of pyrotechnics as proposed by the authors. ${ }^{7)}$ Laser ignition provides much higher thrust than laser ablation. The huge thrust will be useful for the short-time maneuvering of microspacecraft.

In this study, the fundamental thrust performance of a laser ablation thruster is investigated and the ablation processes using a polyvinylchloride (PVC) propellant are clarified. Phipps et al. measured laser ablation thrust using thin polymer films. However, they have not clarified the mechanism of polymer ablation, and this is a definite requirement for practical application and further improvement. First, we measured the fundamental thruster performance of a thick polymer propellant installed on a prototype thruster. Secondly, in order to clarify the mechanism of polymer ablation, we investigated the effect of laser beam intensity and carbon additives in the PVC. In discussions, the relation of performance and laser intensity is explained using a simple photothermal analysis, and the PVC degradation processes using diode lasers is revealed by mass spectroscopy. 


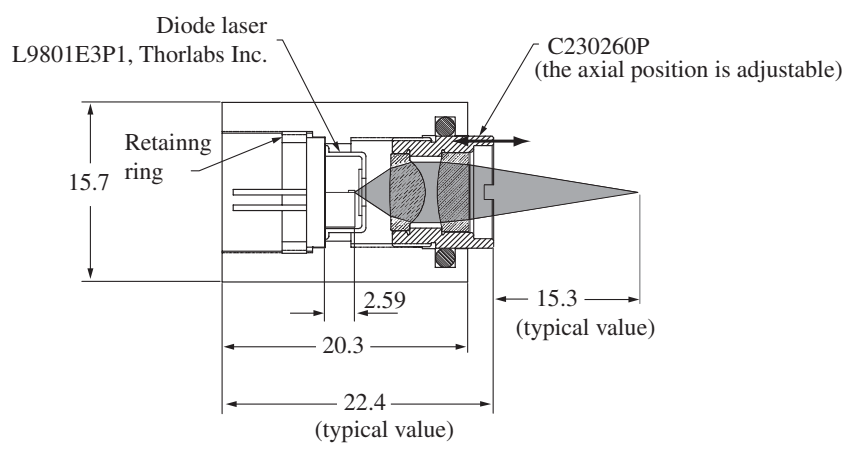

Fig. 1. Schematic diagram and dimensions of the diode laser optical system.

\section{Experimental Setup}

\subsection{Vacuum facilities}

All experiments were performed in a vacuum using a 1.0m-diameter, 1.2-m-long space chamber. The chamber was made of stainless steel and evacuated using two stage pumps: rotary pump and turbo-molecular pump. The base pressures were between $3-7 \times 10^{-3} \mathrm{~Pa}$. The inner pressure was measured using an ionization vacuum gauge.

\subsection{Diode laser optical system}

The optical system employed diode lasers with the maximum beam power of $1 \mathrm{~W}$, a wavelength of $980 \mathrm{~nm}$, and a focal length of $14 \mathrm{~mm}$. The focusing assembly consisted of a 10-mm-diameter lens pair and 15.7-mm-diameter focusing tube optics. A schematic diagram of the diode laser used here is shown in Fig. 1. The focal length was adjusted by changing the position of the lens pair, and the typical length was $15.3 \mathrm{~mm}$, as shown in the figure. The total weight of the diode laser optical system was about $10 \mathrm{~g}$.

\subsection{Laser beam profile}

Generally, a laser beam emitted from a diode laser has an astigmatic elliptical wavefront profile. Figure 2 is a drawing of a typical diode laser beam. The beam has a larger divergence angle perpendicular to the emitting stripe of the diode laser than the angle parallel to it. Then the wavefront of the laser becomes elliptical as shown in the figure. In this study, the perpendicular and parallel axes are referred to as vertical and horizontal axes, respectively. In order to evaluate the effect of laser intensity on laser ablation performance, the beam profile of such an elliptical laser beam was measured

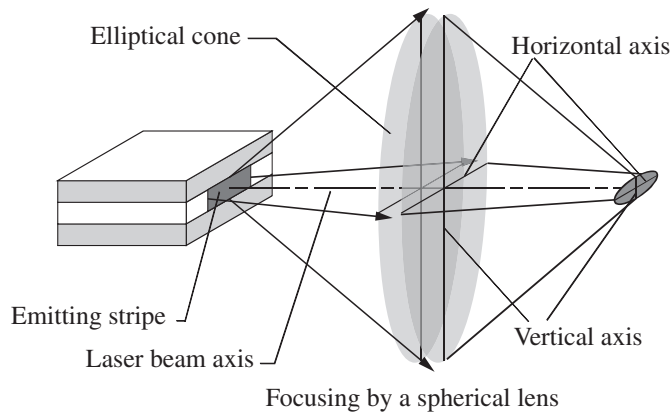

Fig. 2. Drawing of a typical diode laser beam.

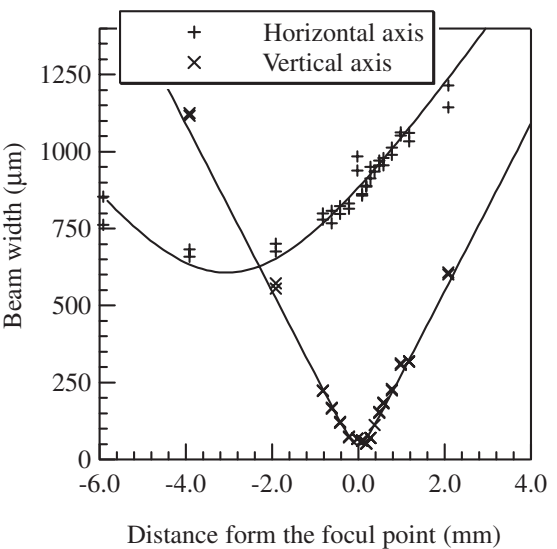

Fig. 3. Measured profiles of beam widths along the beam axis.

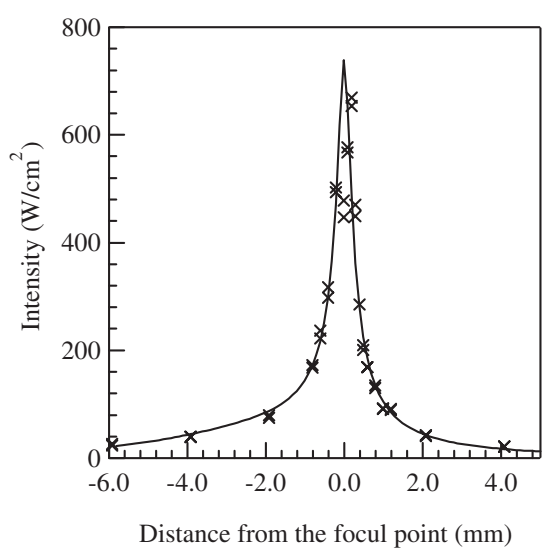

Fig. 4. Laser beam intensity calculated from the beam width profiles.

using a CCD beam profiler. By changing the position of the diode laser against the profiler, a three dimensional beam profile was measured.

The laser beam of a diode laser can be roughly assumed as a Gaussian beam with different beam widths on the vertical and horizontal axes. Then it was assumed that the beam has a Gaussian profile transverse to the beam axis and a hyperbolic profile along that axis. According to the measured beam profile, the parameters of the profiles were determined. Figure 3 shows the change of beam widths along the laser beam axis, where beam width means the $1 / \mathrm{e}^{2}$ power point in the profile.

Figure 4 shows the laser beam intensity calculated from the beam-width profiles (fitted hyperbolic curves). The intensity was defined as incident power over rectangle area (horizontal beam width $\times$ vertical beam width). The intensity has an maximum of $740 \mathrm{~W} / \mathrm{cm}^{2}$ and a full width at half maximum (FWHM) of $0.59 \mathrm{~mm}$.

\subsection{Propellant feeding system}

A cylindrical propellant feeding system was employed to supply a new ablative surface for the laser beam, which has been proposed elsewhere. ${ }^{8)}$ Polymer propellants were applied to a cylinder, and it was installed on a screw fixed on the mounting stage. The laser optical assembly was aligned such that the laser beam was focused on the surface 


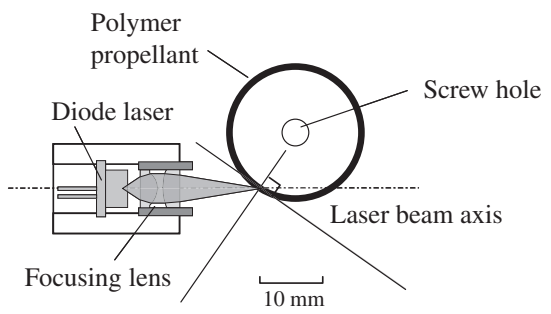

Fig. 5. Configuration of the laser beam and propellant.

of the cylindrical propellant at a specific incident angle. The configurations are shown in Fig. 5. After every shot (laser ablation), the cylinder was rotated upwards or downwards by the screw pitch (DC motor and gearbox were installed above the cylindrical propellant).

\subsection{Thrust and mass measurement}

The impulses generated by the laser ablation jet were measured using a thrust stand, which was described in detail in the literature by Koizumi, $\mathrm{H}$. et al. ${ }^{9}$ It is a horizontally swinging torsional balance with a 30-cm-long arm and 10$\mathrm{cm}$-extension arm to increase the sensitivity. A laser ablation thruster was installed on the extension arm such that the normal vector on the ablation surface was perpendicular to the thrust stand arm. Force-acting period, the period when the thrust is generated, was assumed to be equal to the laser pulse width. This is because the final laser ablation impulse measured showed high proportionality to the laser pulse width. Since the impulse generated by laser ablation is very small, a noise-removing fitting function was used to analyze the displacement. ${ }^{9)}$ The resolution of the thrust stand measurement was $\pm 1.0 \mu \mathrm{Ns}$, and the accuracy of the calibration was $\pm 2 \%$.

In order to evaluate the specific impulse, mass ablated by each laser irradiation $\Delta m$ was estimated from the increase in pressure in the vacuum chamber $\Delta P$ using the equation $\Delta m=C \Delta P$, where $C$ is the proportionality constant. The proportionally constant $C$ was determined by the averaged ablated mass, as determined from the difference in the weight of the propellant before and after 1550-shots experiments divided by the averaged pressure increase during the experiment. The weight difference of the propellant was $29.4 \mathrm{mg}$ after 1550 shots, and the uncertainty of the weight measurement was at most $\pm 5 \%$. The accuracy of individual pressure measurements was $\pm 15 \%$. The specific impulse was evaluated from impulse and ablated mass over 20 shots, for which measurement accuracy was better than $\pm 10 \%$.

\section{Experimental Result}

\subsection{Ablation material dependence}

First, in the atmosphere, several types of propellants were examined for laser ablation: polyvinylchloride (PVC), acrylonitrile butadiene styrene (ABS) polymer, polyoxy methylene (POM), natural rubber (NR), and polymethylmethacrylate (PMMA). Table 1 shows the results. Of those polymer materials, PVC showed the best performance. The emission from the plasma produced by laser ablation was
Table 1. Laser ablation of several polymer materials in the atmosphere.

\begin{tabular}{lcccc}
\hline Name & Color & Shaping & Ablation & Light emission \\
\hline Paper & black & sheet & yes & yes \\
Paper & white & sheet & weak & no \\
PVC & gray & cubic & yes & weak \\
PVC & black & sheet & yes & yes \\
NR & black & sheet & weak & weak \\
ABS & black & cylinder & yes & no \\
POM & black & cylinder & yes & no \\
POM & white & cylinder & no & no \\
PMMA & black & cylinder & weak & no \\
\hline
\end{tabular}

the strongest and the resultant crater was the largest when black-colored PVC was used. However, even if the main component was black PVC, there was large difference between PVCs; this is because of the additives in them. Commercially available polymers usually include several additives for coloring or improving properties.

\subsection{Fundamental thrust performance}

Figures 6 and 7 show the thrust performance using the above-mentioned commercially available black PVC sheet as the propellant. The effect of laser pulse width was investigated at a constant laser power (as a result, incident laser energy changed). The beam power was set to $280 \mathrm{~mW}$, which is adequate power for power-limited microspacecraft (the available power would be a maximum of $1 \mathrm{~W}$ ). The impulse was proportionally increased up to $8.1 \mu \mathrm{Ns}$ with the increase of the pulse width up to $800 \mathrm{~ms}$. The ablated mass was also increased up to $6.0 \mu \mathrm{g}$.

The proportionality of impulse and mass to energy means the constant specific impulse and momentum coupling coefficient. The momentum coupling coefficient, abbreviated as $C_{\mathrm{m}}$, is defined as the impulse provided by laser ablation over the incident energy (equal to the thrust-to-power ratio). The specific impulse and coupling coefficient are shown in Fig. 7. They are almost constant against the laser energy, with the averaged value of $134 \mathrm{~s}$ and $42 \mu \mathrm{Ns} / \mathrm{J}$, respectively. Both the Isp and $C_{\mathrm{m}}$ in Fig. 7 show slight increases as the laser pulse decreases. This has also been confirmed by Phipps et al. ${ }^{5,6)}$

\subsection{Effect of laser intensity}

In order to evaluate the influence of laser intensity, laserablated mass was measured while changing the intensity. The position of the target was shifted around the focal point. Changing the position resulted in different laser intensities according to the measured beam profile. The relation of the target surface position from the focal point and the ablated mass is shown in Fig. 8, where laser intensity was calculated from the position using the relation of Fig. 4. The distance from the optical system to the target surface is uncertain because of the oblique surface of cylindrical propellant. The associated error was a maximum of $\pm 1.0 \mathrm{~mm}$.

Figure 8 shows that the laser-ablated mass gradually changed with the position, whereas the laser intensity abruptly changed around the focal point, thereby showing that laser-ablated mass has little dependence on laser 


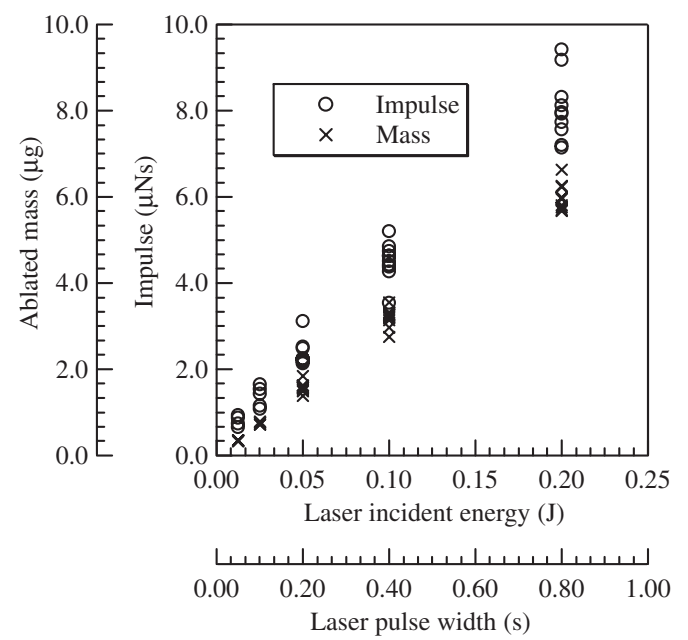

Fig. 6. Ablation jet impulse and mass using commercially available black $\mathrm{PVC}$ as the propellant.

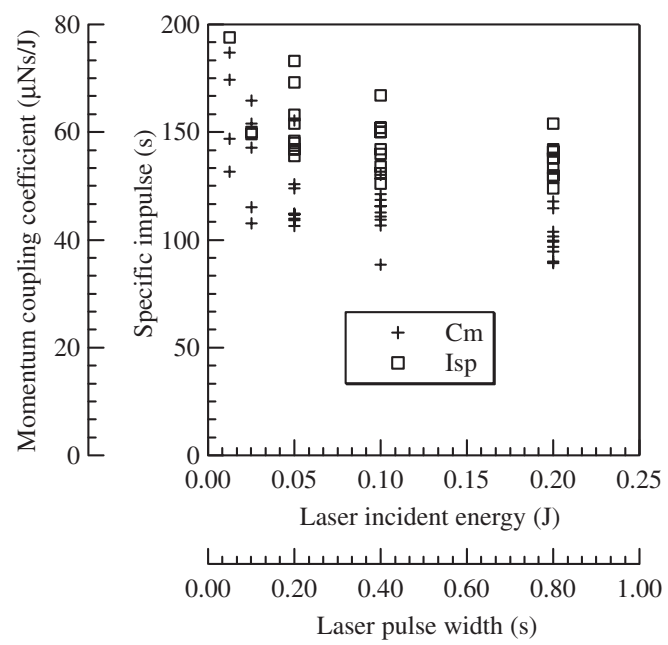

Fig. 7. Specific impulse and momentum coupling coefficient using commercially available black PVC as the propellant.

intensity, at least in the vicinity of the focal point. From an engineering viewpoint, weak dependence of the intensity on the ablated mass, namely the performance, enables the course alignment for the optical system.

\subsection{Effect of carbon additives}

The effect of the carbon additives in PVC was investigated. It should be noted that black-colored material showed the best performance. This can be easily understood by the fact that the wavelength of the laser beam was close to visible light. Hence, the absorption coefficient, shown as color, drastically affects thruster performance. Three PVC cylinders with carbon densities of $0,2.5$, and $5.0 \%$ were prepared and their thrust performances were measured. A picture of those PVC cylinders is shown in Fig. 9. PVC with $0 \%$ carbon is almost transparent and the others are opaque (black).

As the carbon density in PVC increased, a dramatic increase of the performance was observed. The measured thrust performances are shown in Figs. 10 and 11. The

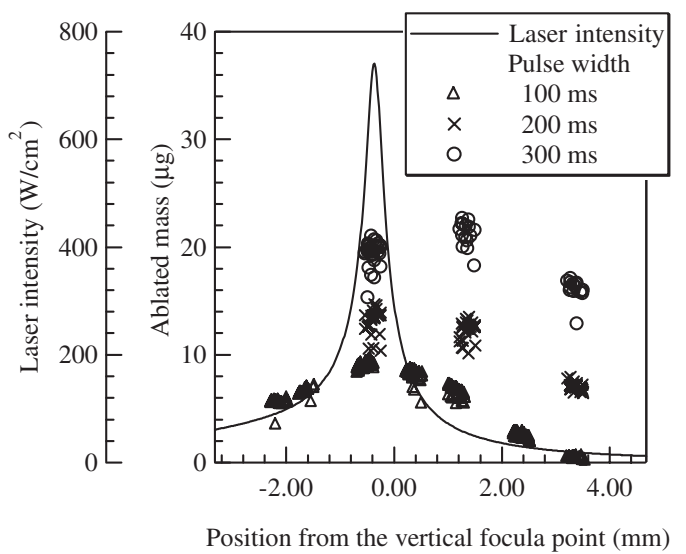

Fig. 8. Effect of the laser intensity on the ablated mass.

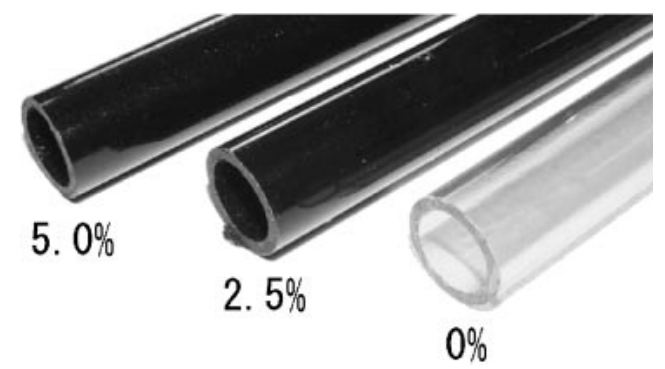

Fig. 9. Picture of the PVC cylinders with different doped carbon densities: $0,2.5$ and $5,0 \%$.

measurement was carried out for 2.5 and $5.0 \%$ carbon PVC $(0 \%$ carbon PVC showed no reaction to laser focusing). PVC with $5.0 \%$ carbon showed a coupling coefficient 5.9 times greater than that of $2.5 \%$. In contrast, the specific impulse for $5.0 \%$ carbon was 1.7 times that of $2.5 \%$. Therefore, the increase in impulse was mainly caused by the increase in ablated mass. As a result, using PVC with $5.0 \%$ carbon as the propellant obtained the thrust performance of: specific impulse, $104 \mathrm{~s}$ and coupling coefficient, $152 \mu \mathrm{Ns} / \mathrm{J}$. These values are higher than using the commercially available PVC shown in Fig. 7. Especially, the coupling coefficient was increased threefold from 42 to $152 \mu \mathrm{Ns} / \mathrm{J}$.

\section{Discussions}

\subsection{Variations of impulse}

The precise positioning of microspacecraft requires not only small thrust, but also accuracy (resolution). Precise attitude or translation control requires an accuracy of micro-Newton order. For instance, LISA, ${ }^{10)}$ which comprises a laser interferometer, requires extreme accuracy; 5$30 \mu \mathrm{N}$ thrust with a resolution of less than $0.1 \mu \mathrm{N}$. Thrust measurement of the above laser ablation thruster showed the linearity of the thrust to the laser pulse width, and the thrust (impulse) is predicted by the width. In the experiment using commercially available PVC (Fig. 6), the relation was $I(\mu \mathrm{Ns})=9.59 \times$ pulse $(\mathrm{s})+0.48$. The standard deviation 

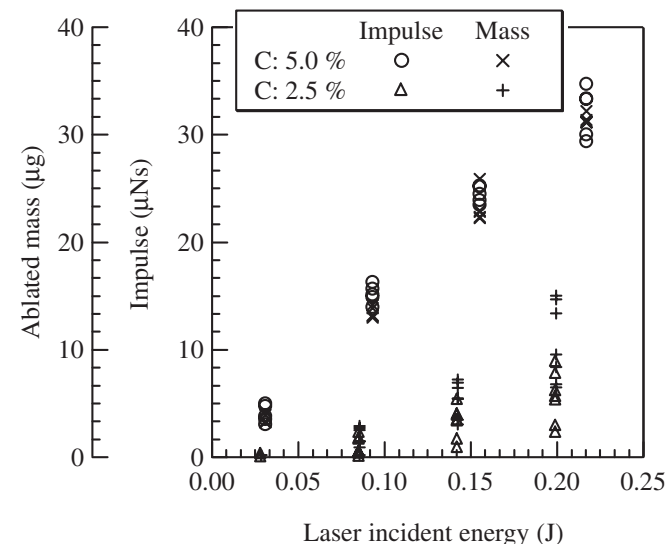

Fig. 10. Ablation jet impulse and mass using custom-made PVCs with carbon additives of 2.5 and $5.0 \%$.

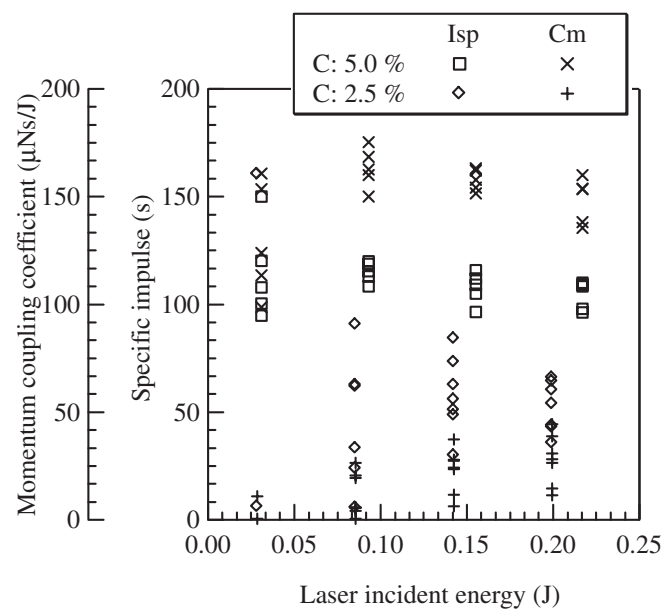

Fig. 11. Specific impulse and momentum coupling coefficient using custom-made PVCs with carbon additives of 2.5 and $5.0 \%$.

from that proportionality was $0.48 \mu \mathrm{Ns}$ with an impulse range of 1 to $8 \mu \mathrm{Ns}$. In the experiment using an other material with a higher coupling coefficient (Fig. 10), the standard deviation was $1.5 \mu \mathrm{Ns}$ with an impulse range of 5 to $35 \mu \mathrm{Ns}$.

\subsection{Thermal analysis of laser ablation}

To clarify the ablation mechanism by diode laser irradiation, a photothermal ablation model is applied for the laser ablation processes. Here, it is assumed that the laser beam irradiated on the ablative propellant is converted from photo energy to thermal energy by absorption. This is because the laser intensity of a diode laser is not sufficiently high enough to cause a photochemical reaction. Hence, the beam behaves as a heat source in the ablative material, and it is analyzed as a heat conduction problem.

The laser ablation processes are assumed to be constructed by the following two regimes. First, the laser beam is absorbed in the ablative material and all the energy is used to raise the inner temperature. Second, after the maximum temperature (generally surface temperature) reaches the boiling or degrading point, ablation proceeds with a constant temperature profile inside the solid body. In the second

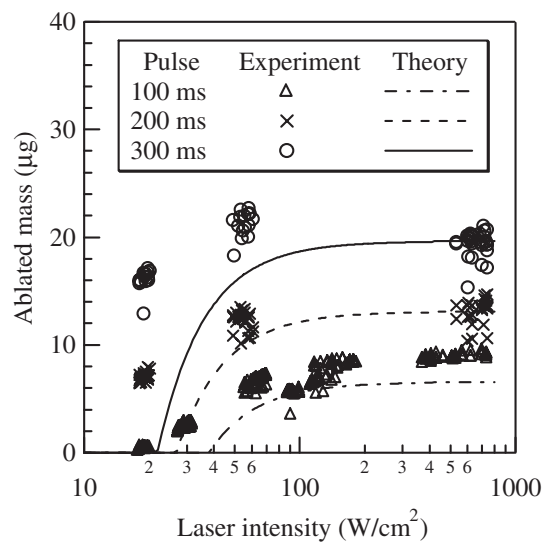

Fig. 12. Relation of the laser intensity and ablated mass.

process, the laser energy is used to ablate the material at the surface and maintain the shape of the temperature profile, which is called "stationary ablation". Actually, between the two processes there is a transition process to reach steady ablation, but in the simple model dealt with here, the transition was neglected.

In the simplest case, let us consider that the laser absorption length is short enough. It would be easily accomplished using a material with high carbon density. Hence, the heat source is involved in the boundary condition, and the governing equation is:

$$
\begin{aligned}
\frac{\partial T}{\partial t}=\kappa \frac{\partial^{2} T}{\partial x^{2}} \quad \text { for } x>0 \\
\text { i.c. } \quad T(x, 0)=T_{\infty} \quad \text { for } x>0 \\
\text { b.c. } \quad T_{x}(0, t)=q / K, \quad T_{x}(\infty, t)=T_{\infty},
\end{aligned}
$$

where $T$ is the temperature, $t$ is the time, $x$ is the position from the ablation surface, $\kappa$ is the thermal diffusivity, $K$ is the thermal conductivity, and $q$ is the incident heat flux. The maximum temperature obviously exists at $x=0$, and the boiling point $T_{\mathrm{b}}$ is reached when:

$$
t_{\mathrm{b}}=\frac{1}{\kappa}\left(\frac{\sqrt{\pi}}{2} \frac{K\left(T_{\mathrm{b}}-T_{\infty}\right)}{q}\right)^{2}
$$

In general, polymers have no clear boiling point and the solid-state polymer gradually changes into the gas phase. Hence, the degradation point should be used rather than boiling point. One dimensional stationary ablation has the recession velocity $v$ of:

$$
v=\frac{q}{\rho\left(\Delta h+c_{\mathrm{p}}\left(T_{\mathrm{b}}-T_{\infty}\right)\right)},
$$

where $\rho$ is the polymer density, $\Delta h$ is the heat of ablation, and $c_{\mathrm{p}}$ is the specific heat. Here, it should be noted that the stationary ablation rate is independent of the heat source profile once it reaches steady-state. As a result, the laser-ablated mass $m(t)$ can be expressed as:

$$
m(t)=\left\{\begin{array}{cc}
0, & t<t_{\mathrm{b}} \\
\rho v A\left(t-t_{\mathrm{b}}\right), & t \geq t_{\mathrm{b}}
\end{array},\right.
$$




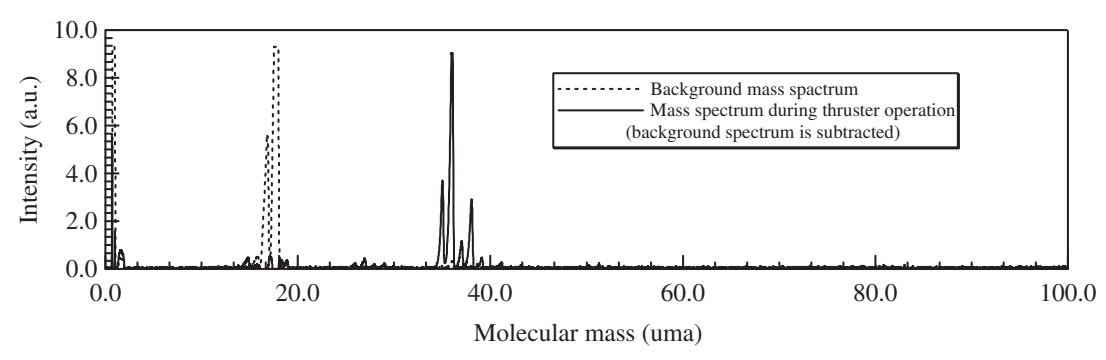

Fig. 13. Mass spectrum during the repulsive laser ablations.

where $A$ is the area irradiated by the laser beam. This means that mass ablated by laser irradiation mainly depends on the total incident energy and little on the intensity. The intensity only has an effect when ablation starts.

Figure 12 is a re-drawing of the data of Fig. 8 as the relation of laser intensity and ablated mass, where the theoretical curves of ablated mass in Eq. (5) are drawn as a function of intensity $q$. Here, the heat of fusion was experimentally estimated. As mentioned, polymer generally shows a vague degradation point and it is difficult to determine the value. Therefore the heat of ablation was determined as $4.6 \mathrm{MJ} /$ $\mathrm{kg}$ from the ratio of ablated mass to laser pulse width at a high laser intensity $\left(>300 \mathrm{~W} / \mathrm{cm}^{2}\right)$. Other physical and thermal properties used here are density: $1350 \mathrm{~kg} / \mathrm{m}^{3}$, thermal conductivity: $0.21 \mathrm{~W} / \mathrm{mK}$, molecular, specific heat: $952 \mathrm{~J} /$ $\mathrm{kg} / \mathrm{K}$, and degradation point: $553 \mathrm{~K}$. The theoretical curves show the qualitative behavior of the ablation. The ejected mass increases with the laser intensity in a higher intensity regime, and it becomes saturated in the higher intensity regime. This means that the ablated mass shows little dependence on the intensity if the intensity is high enough, and this tendency agrees with the theoretical prediction of Eq. (5). However, the predicted beginning of ablation is slower than the measured mass, in spite of disregarding the transition regime here. The rising time is determined by density, specific heat, thermal conductivity, and laser intensity. Uncertainty included in the thermal properties and intensity would cause that difference. Especially, the intensity would include large error, and additionally affect the rising time quadratically.

\subsection{Mass spectroscopy}

To investigate the degradation processes on PVC material, a mass spectroscopy of the exhaust plume was performed using a quadruple mass spectrometer. Figure 13 shows the measured mass spectrum during the repulsive operation of the thruster (PVC with 5\% carbon was used). The mass spectrum of residual gases (background) was also measured, where intense peaks of 1,17 , and 18 attributed to water $\left(\mathrm{H}_{2} \mathrm{O}\right)$ were observed. The background spectrum was subtracted from the spectrum during the thruster operation. Therefore, the contributions from residual gases were eliminated and only ablated gas from the PVC remained. The spectrums were searched in the range of 1-100 amu.

In Fig. 13, four strong peaks of 35 to 38 are identified. These peaks correspond to $\mathrm{HCl}$ and $\mathrm{Cl}$ with those isotope from other literature. ${ }^{11)}$ The ratio of measured peaks and
Table 2. Comparison of the experimentally measured peaks and abundance ratio of $\mathrm{Cl}$ and $\mathrm{HCl}$ isotope.

\begin{tabular}{cccc}
\hline $\begin{array}{c}\text { Mass number } \\
\text { (u.m.a) }\end{array}$ & $\begin{array}{c}\text { Experimental } \\
\text { result }\end{array}$ & $\begin{array}{c}\text { Chemical } \\
\text { composition }\end{array}$ & $\begin{array}{c}\text { Fraction of } \\
\text { isotope }\end{array}$ \\
\hline 35 & 0.72 & ${ }^{35} \mathrm{Cl}$ & 0.76 \\
37 & 0.28 & ${ }^{37} \mathrm{Cl}$ & 0.24 \\
36 & 0.86 & ${ }^{1} \mathrm{H}^{35} \mathrm{Cl}$ & 0.76 \\
38 & 0.14 & ${ }^{1} \mathrm{H}^{37} \mathrm{Cl}$ & 0.24 \\
\hline
\end{tabular}

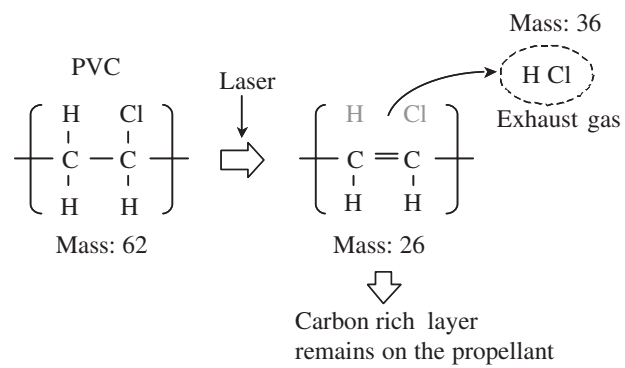

Fig. 14. Dehydrochlorination of the PVC with the subsequent formation of conjugated double bonds.

abundance ratio of $\mathrm{HCl}$ and $\mathrm{Cl}$ are listed in Table 2, which shows good agreement. Such strong emissions of $\mathrm{HCl}$ from PVC by laser or heating is in agreement with many other studies. ${ }^{11-13)}$ They have shown that the thermal degradation of PVC proceeds in the following two stages. The first stage proceeds in the temperature range of 470 to $640 \mathrm{~K}$. It corresponds to dehydrochlorination with the subsequent formation of conjugated double bonds, as shown in Fig. 14. The second stage proceeds in the range of $640-770 \mathrm{~K}$, which is the scission of polyene sequences formed during the first stage. The results of mass spectroscopy show that the ablated gas generated by the a diode laser in this study would come from the first stage reaction.

The laser ablation of PVC with carbon additives can be summarized as follows. Fist, laser irradiation is absorbed by the doped carbon, not by the PVC itself. The absorbed energy is delivered to the PVC by thermal conduction. When the temperature of the PVC reaches 470 to $640 \mathrm{~K}$, polymer degradation starts and $\mathrm{HCl}$ is emitted by dehydrochlorination. The preferential degradation of $\mathrm{HCl}$ at low temperatures will cause the best performance of PVC among the several polymer materials. 


\section{Conclusion}

In this study, the fundamental thrust performance of a diode laser microthruster was investigated and the following conclusions were obtained. Successful operations of the laser ablation thruster were verified using a polyvinylchloride propellant. The thrust provided by laser ablation was proportional to the laser pulse width. The momentum coupling coefficient ranged from 40 to $150 \mu \mathrm{Ns} / \mathrm{J}$, corresponding to the ablative material. The impulse variation was a maximum of $0.5 \mu \mathrm{Ns}$, and it was shown that the thruster gives minute thrust resolution for tight attitude or translation control. Polyvinylchloride targets doped with carbon were examined to investigate the effect of carbon content. As a result, carbon density drastically affected the performance, momentum coupling coefficient. Laser beam intensity was changed while maintaining laser power, but it hardly affected the resulting impulse in spite of a large change in intensity (200$750 \mathrm{~W} / \mathrm{cm}^{2}$ ). This is attractive from the engineering viewpoint, and that behavior was reproduced using a simple photo-thermal analysis. A mass spectroscopy of the exhausted gas showed that the dominant reaction of the PVC ablation was dehydrochlorination in the range of 470-600 K, which would cause the best performance of PVC.

\section{References}

1) Mueller, J.: Thruster Options for Microspacecraft: A Review and Evaluation of Evaluation of State-of-the-Art and Emerging Technologies, Micropropulsion for Small Spacecraft, American Institute of Aeronautics and Astronautics, Reston, VA, 2000, Chapter 3.
2) Mukerjee, E. V., Wallace, A. P., Yan, K. Y., Howard, D. W., Smith, R. L. and Collins, S. D.: Vaporizing Liquid Microthruster, Sensors and Actuators, A 83 (2000), pp. 231-236.

3) Lewis, D. H., Janson, S. W., Cohen, R. B. and Antonsson, E. K.: Digital Micropropulsion, Sensors and Actuators, A80 (2000), pp. 143-154.

4) Burton, R.L. and Turchi, P.J.: Pulsed Plasma Thruster, J. Propul. Power, 14 (1998), pp. 716-735.

5) Phipps, C. and Luke, J.: Diode Laser-Driven Microthrusters: A New Departure for Micropropulsion, AIAA J., 40 (2002), pp. 310-318.

6) Phipps, C., Luke, J., Lippert, T., Hauer, M. and Wokaun, A.: Micropropulsion Using a Laser Ablation Jet, J. Propul. Power, 20 (2004), pp. 1000-1010.

7) Koizumi, H., Inoue, T., Nakano, M. and Arakawa, Y.: Dual Propulsive Mode Microthruster Using a Diode Laser, J. Propul. Power, 21 (2005), pp. 1133-1136.

8) Koizumi, K., Inoue, T., Kojima, K., Mori, K., Komurasaki, K. and Arakawa, Y.: Microthruster Experiment Using a Diode Laser, AIAA Paper-2003-4568.

9) Koizumi, K., Komurasaki, K. and Arakawa, Y.: Development of Thrust Stand for Low Impulse Measurement from Microthrusters, Rev. Sci. Instrum., 75 (2004), pp. 3185-3189.

10) Danzmann, K. and LISA Study Team: Lisa: Laser Interferometer Space Antenna for the Detection and Observation of Gravitational Waves, 2nd ed., Max-Planck Inst. fur Quantenoptik, Garching, Germany, 1998.

11) Castro, C.S.C., Azevedo, A.C.P., Monteiro, E.E.C., Phinho, R. R. and Souza, G.G.B.: Gas Emission from Chlorinated Polymers Induced by Synchrotron Radiation, Polymer Degradation and Stability, $\mathbf{7 1}$ (2001), pp. 233-242.

12) Miranda, R., Yang, J., Roy, C. and Vasile, C.: Vacuum Pyrolysis of pvc I. Kinetic Study, Polymer Degradation and Stability, 64 (1999), pp. 127-144.

13) Kokai, F., Koga, Y., Kakudate, Y., Kawaguchi, M., Fujiwara, S., Kubota, M. and Fukuda, K.: Laser-Ionization Mass-Spectrometric Studies on Laser Ablation of a Nitrogen-Rich Polymer at $532 \mathrm{~nm}$ and 1064 nm, Appl. Phys. A, 59 (1994), pp. 299-304. 